\title{
An intravascular coronary plaque elasticity reconstruction method using limited depth penetration ultrasound signals
}

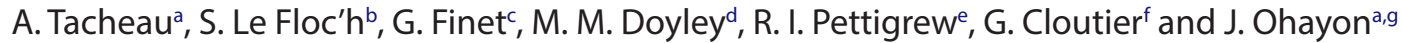 \\ aLaboratory TIMC-IMAG/DyCTiM2, UJF, CNRS UMR 5525, Grenoble, France; 'baboratory LMGC, CNRS UMR 5508, University of Montpellier II, \\ Montpellier, France; ' CHospices Civils de Lyon and Claude Bernard University Lyon1; INSERM Unit 886, Lyon, France; ${ }^{d}$ Department of Electrical \\ and Computer Engineering, University of Rochester, Rochester, NY, USA; ${ }^{N}$ National Institute of Diabetes Digestive and Kidney Diseases, National \\ Institutes of Health, Bethesda, MD, USA; 'Laboratory of Biorheology and Medical Ultrasonics, CRCHUM, Montréal, Canada; ${ }^{9 U n i v e r s i t y ~ S a v o i e ~}$ \\ Mont-Blanc, Polytech Annecy-Chambéry, Le Bourget du Lac, France
}

KEYWORDS Modulography; linear elasticity; inverse problem; vulnerable plaques; coronary disease

\section{Introduction}

Vulnerable atherosclerotic plaque (VP) rupture is the leading cause of acute coronary syndrome, myocardial infarction and stroke in the Western world (Go et al. 2013). Although past studies have identified fibrous cap thickness $\left(\mathrm{Cap}_{\text {thick }}\right)$ as the primary predictor of coronary plaque rupture (Virmani et al. 2006), biomechanical studies have recognized peak cap stress (PCS) as an additional key predictor of plaque disruption (Ohayon et al. 2008). Quantification of PCS amplitude requires not only an accurate description of plaque morphology but also a precise knowledge of the mechanical properties of plaque components. An early and accurate determination of these properties remains an essential step to implement preventive therapeutic strategies.

A previous approach (named iMOD for imaging modulography) has been conducted by our group to reconstruct the Young's modulus map (i.e. modulogram) from intravascular ultrasound (IVUS) strain-elastogram (Le Floc'h et al. 2009). However, one major limitation of such a method is the need to accurately estimate the strain field in the entire lesion, which may be often difficult when using HD-IVUS techniques with limited depth penetration signals (Kobayashi et al. 2014). Therefore, an extended iMOD approach (E-iMOD), based on the continuum mechanics theory prescribing the strain field in the limited atherosclerotic endoluminal region, was designed and successfully applied to coronary lesions of patients imaged in vivo with IVUS.

\section{Methods}

\subsection{Population}

Seven plaques geometries, acquired from patients with stable angina and scheduled to undergo percutaneous coronary intervention at the Hospital of Cardiology and Pneumology of Lyon, were used to initiate the finite element (FE) analysis. The IVUS echogenicity aspects were used to characterize VP components.
The digitized contours obtained with ImageJ were imported into MATLAB and then transferred in the FE software Comsol (Structural Mechanics Module, version 3.5, Comsol, France).

\subsection{Plaque strain and displacement reconstructions}

The mechanical properties of the fibrous regions, calcified inclusions and soft necrotic cores were modelled as isotropic and quasi-incompressible media (Poisson's ratio $\nu=0.49$ ) with Young's moduli $E_{\text {fbrosis }}=800 \mathrm{kPa}, E_{\text {calcified }}=5000 \mathrm{kPa}$ and $E_{\text {core }}=5 \mathrm{kPa}$ (Finet et al. 2004). A pressure gradient $\Delta P$ of $1 \mathrm{kPa}(7.5 \mathrm{mmHg})$ occurring between two successive frames of recorded IVUS sequence was assumed. Displacement and radial strain fields $\left(\vec{u}^{\text {meas }}, \varepsilon_{r r}^{\text {meas }}\right)$ were obtained by performing static FE computations on the VP geometries under the assumption of plane strain. The performance of the proposed elasticity reconstruction method E-iMOD was tested with these input displacement and radial strain fields.

\subsection{Inverse problem: elasticity reconstruction}

The theoretical framework of this method is based on the one proposed previously by our group (Le Floc'h et al. 2009). Briefly, considering two successive frames of the IVUS sequence, we applied the iMOD technique, which involves three successive steps: (i) the computation of a pseudo-gradient elasticity map, (ii) the dynamic segmentation procedure that makes use of the previous step results to extract the inclusions' contours, and (iii) the mathematical optimization procedure that provides the estimated Young's moduli of detected inclusions and surrounding tissue.

\section{Results}

Figure 1 illustrates the performance of E-iMOD algorithm to detect soft necrotic core. Based on the results obtained on our 

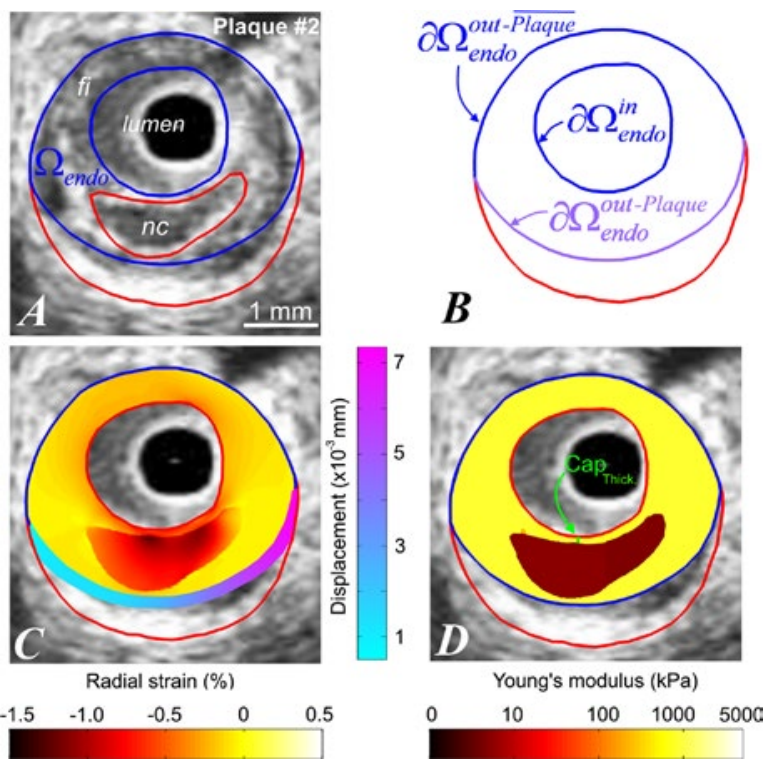

Figure 1. (A)-(B) Geometry extracted from IVUS acquisition, in blue the endoluminal contours (C) Radial strain-elastogram and amplitude of the displacement vector applied. (D) Computed modulogram.

Notes: nc: necrotic core. fi: fibrous region.

entire population $(n=7)$, we show that (1) E-iMOD method identified reasonably Young's moduli of the necrotic core and fibrous region with mean values of $5.41 \pm 0.51 \mathrm{kPa}$ and $790.61 \pm 23.28 \mathrm{kPa}$ instead of 5 and $800 \mathrm{kPa}$, respectively, and (2) $\mathrm{Cap}_{\text {thick }}$ amplitudes are with minimal and maximal relative errors close to $-2.7 \%$ (corresponding to a computed Cap thick $_{\text {of }} 91 \mu \mathrm{m}$ for a theoretical value of $93 \mu \mathrm{m}$ ) and $+64.6 \%$ (corresponding to a computed Cap thick $_{\text {of }} 68 \mu \mathrm{m}$ instead of $41 \mu \mathrm{m}$ ), respectively.

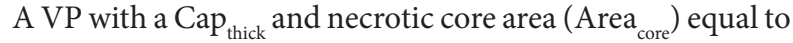
$93 \mu \mathrm{m}$ and $1.36 \mathrm{~mm}^{2}$, respectively, was used to study the influence of white noise (Le Floc'h et al. 2009) on Young's modulus and plaque morphology reconstructions. The performed simulations showed that E-iMOD method was able to highlight the VP morphology when introducing significant white noise on both radial strain and displacement fields (up to $3 \mathrm{~dB}$ ). The

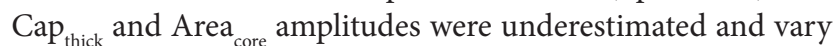
from $87.19 \pm 7.51 \mu \mathrm{m}$ and $1.32 \pm 0.03 \mathrm{~mm}^{2}$ with a white noise of $18 \mathrm{~dB}$ to $82.75 \pm 5.63 \mu \mathrm{m}$ and $1.18 \pm 0.28 \mathrm{~mm}^{2}$ with high white noise $(3 \mathrm{~dB})$, respectively. The E-iMOD method identified reasonably mean Young's moduli of the necrotic core and fibrous region with both maximal relative error lower than $20 \%$. According to this noise study, the algorithm still gave reasonable results when introducing significant white noise amplitude.

\section{Conclusions}

This study demonstrates the potential of the revisited IVUS E-iMOD modulography technique to detect and quantify both the mechanical properties and the morphologies of VPs when considering HD-IVUS catheters with limited depth penetration.

\section{Acknowledgement}

Antoine Tacheau held a doctoral fellowship from University Grenoble-Alpes, France (AGIR 2013). Funding of this project was provided by a grant from la Région Rhône-Alpes 20142015.

\section{References}

Finet G, Ohayon J, Rioufol G. 2004. Biomechanical interaction between cap thickness, lipid core composition and blood pressure in vulnerable coronary plaque: impact on stability or instability. Coron Artery Dis. 15:13-20.

Go AS, Mozaffarian D, Roger VL, Benjamin EJ, Berry JD, Borden WB, Bravata DM, Dai S, Ford ES, Fox CS, et al. 2013. Executive summary: heart disease and stroke statistics 2013 update: a report from the American Heart Association. Circulation. 127:143-152.

Kobayashi Y, Kitahara H, Tanaka S, Nakagawa K, Okada K, Otagiri K, Yock P, Fitzgerald P, Ikeno F, Honda Y. 2014. TCT-363 precision of a novel high-definition $60 \mathrm{MHz}$ IVUS in quantitative measurement. J Am Coll Cardiol. 64:B105B106.

Le Floc'h S, Ohayon J, Tracqui P, Finet G, Gharib AM, Maurice RL, Cloutier G, Pettigrew RI. 2009. Vulnerable atherosclerotic plaque elasticity reconstruction based on a segmentation-driven optimization procedure using strain measurements. IEEE Trans Med Imaging. 28:1126-1137.

Ohayon J, Finet G, Gharib AM, Herzka DA, Tracqui P, Heroux J, Rioufol G, Kotys MS, Elagha A, Pettigrew RI. 2008. Necrotic core thickness and positive arterial remodeling index: emergent biomechanical factors for evaluating the risk of plaque rupture. Am J Physiol Heart Circ Physiol. 295:H717H727.

Virmani R, Burke AP, Farb A, Kolodgie FD. 2006. Pathology of the vulnerable plaque. J Am Coll Cardiol. 47(8 Suppl): C13-C18. 